\title{
The withdrawal of treatment is still treatment
}

\author{
Joel B. Zivot, MD
}

Received: 13 July 2014/ Accepted: 18 July 2014/Published online: 5 August 2014

(C) Canadian Anesthesiologists' Society 2014

In the paper by Hawryluck et al. ${ }^{1}$ examining Cuthbertson v. Rasouli, the suggestion is made that the Supreme Court of Canada (SCC) changed the rules of engagement around end-of-life decisions when doctors and patients disagree. In fact, the SCC did not disturb prior decisions on the role of experts and the jurisdiction of the courts and restated what has always been the case, i.e., the withdrawal of treatment is treatment and, as such, requires consent. The news for physicians is good. The court neither overreached nor tied the hands of physicians when it cited "health-related purpose". Important medical ethical principles remain in place and will continue to guide complex treatment choices as doctors and patients struggle to do the right thing at the end of life.

The details of Hawryluck et al. warrant further discussion. A deeper analysis is required concerning the standard of care and the concept of medical benefit and what the SCC intends by citing "health-related purpose". Future legal actions with the same question as that contained within Cuthbertson v. Rasouli will, and should always, be decided in the same manner as Cuthbertson $v$. Rasouli. Hawryluck et al. also misunderstand the role of the Ontario Consent and Capacity Board (CCB). An examination and understanding of the medical ethical principles at stake will provide the framework for a way forward.

Standard of care describes the common medical practice of prudent physicians at a particular time and in a particular circumstance. It is a majority opinion that firms up before the courts but loosens up at the bedside. The standard of

J. B. Zivot, MD ( $\varangle)$

Department of Anesthesiology, Emory University, 201 Dowman

Dr, Atlanta, GA 30322, USA

e-mail: joel.zivot@emoryhealthcare.org care and the obligation of the physician have always fallen strongly in favour of preserving life. Can the standard of care alone be a reason to discontinue treatment? If the SCC had considered the standard of care, it is false to suggest that it would have carried the day and found in favour of the doctors. The court has never conceded that only doctors decide the extent of medical expertise. The court has the power to adjudicate on issues that it considers within its authority to do so and has made this point before. In 2001, in Walker Estate v. York Finch General Hospital et al., ${ }^{2}$ the SCC found that a Red Cross pamphlet given to potential blood donors to discourage donation in groups at high risk of infection with HIV was inadequate despite evidence to the contrary by two medical experts. This case showed that the court would not allow doctors to decide on questions that do not require special skills or expertise. If an issue is outside of the knowledge of the courts, it will rely on experts, but it is hubris to suggest that the question of a life worth living is a matter of expert medical opinion. Only the individual knows the value of his or her own life.

In the use of the term "health-related purpose", the SCC points out that the Ontario legislature sought to make clear the need for consent for any treatment. Health-related purpose unambiguously affirms that the withdrawal of treatment is treatment and that all treatment requires consent. If the capacity to consent is at issue, the proper place for disputes regarding consent reside within the CCB. The CCB was originally created to review involuntary committal under the Mental Health Act. ${ }^{\mathrm{A}}$ The CCB panel traditionally consists of lawyers, laypersons, and a psychiatrist. In involuntary committal, a psychiatrist on

\footnotetext{
$\bar{A}$ Michael Bay, Personal Communication, July 10, 2014, Mr. Bay established Ontario's Consent and Capacity Board and served as its Chair and CEO from 1995 to 2002.
} 
the panel is necessary to act in place of the treating psychiatrist who had initiated the committal action. In the case of an end-of-life conflict initiated by a doctor, where no substitute decision maker is in place, the board now plays the role of the patient so far as giving consent to withdraw life support. Adding doctors trained in critical care to the board, as Hawryluck et al. suggest, misunderstands the role of the CCB. The CCB is not concerned with standard of practice, and it should not necessarily be any more knowledgeable than a layperson would be. A physician cannot unilaterally decide to overrule a decision by a competent patient or their substitute decision makers (SDM) on matters relating to autonomy, of which withholding treatment is one. If a group of physicians agree on a treatment and offer it as the standard of care, it is no more compelling than if an individual physician recommended it. The standard of care was never intended to be an ad populum tactic to force compliance in competent but unwilling patients.

A common argument advanced as justification for withdrawal of life support concerns intractable suffering. Suffering is bound up in the concept of "benefit", which is derived from the Latin, benefactum, meaning "good deed". A good deed is not a unilateral action. "Benefit" is decided by a contract; there must be offer, acceptance, and consideration. A patient must agree that the deed is in fact "good" and autonomy means the patient decides what is tractable or not. In the case of suffering, the standard of care becomes a value question. Though we might agree that we are against suffering, claims of suffering made by the outward observation of third parties are not necessarily objective. Suffering is subjective and therefore relative. Suffering is often tied to the concept of futility; however, futility claims are tautological and have no consensus in the details. Let us not pretend that physicians are objective about withdrawal of life support. In truth, the standard of care is far from monolithic. Physician decision-making is not free from bias, and studies have shown that physician end-of-life decisions often depend on the religious beliefs of the physician and the geographic location of the practice. $^{3}$

Defensible ethical theories are guided, but not governed, by principles alone. The virtue of caring can free the physician from narrow concepts contained within codes of ethics. The practice of medicine at the bedside is the balance between our reason and our desire. What do patients and families mostly need from us? The Aristotelian concept of phroneis, or practical wisdom, applies here. Discerning physicians not only know that a principle applies but also know how to apply it in the circumstance at hand. The experienced physician creates a bond of trust with the patient, and the trust guides the treatment. Rarely do patients and families take positions that defy all reason. When trust is broken, every disagreement is insurmountable. We must prevent it from reaching that stage by taking a step back, (i.e., taking time out), letting emotions subside, and then returning to consider the recommendations. End-of-life decisions are the biggest decisions that people make. We should give patients the time they need. Critical care specialists know that death comes to everyone despite our efforts. When a family consents to a withdrawal of treatment, this is not a victory for the standard of care, as Hawryluck et al. would have it understood, and if it takes some patients or their SDM longer to accede to the inevitability of death, so be it.

\section{L'arrêt d'un traitement est aussi un traitement}

Dans l'article de Hawryluck et coll. ${ }^{1}$ examinant l'affaire Cuthbertson contre Rasouli, il est suggéré que la Cour suprême du Canada (CSC) a modifié les règles entourant les prises de décision concernant la fin de vie lorsque médecins et familles de patients ne sont pas d'accord. En fait, la CSC n'a pas bouleversé de décisions antérieures sur le rôle des experts et la compétence des tribunaux; elle a réaffirmé ce qui a toujours été le cas : l'arrêt d'un traitement est un traitement et, en tant que tel, nécessite un consentement. Cette décision est une bonne nouvelle pour les médecins. La Cour n'a pas outrepassé son rôle en invoquant un « objectif lié à la santé » et elle n'a pas, non plus, lié les mains des médecins. Les principes éthiques importants de la médecine restent intacts et continueront à guider les choix thérapeutiques complexes dans lesquels se débattent médecins et patients lorsqu'il s'agit de prendre la meilleure décision qui soit en fin de vie.

Les détails de l'article de Hawryluck et coll. justifient de pousser la discussion plus loin. Une analyse plus approfondie concernant les normes de soins et le concept de bénéfice clinique est nécessaire, ainsi qu'un examen minutieux de ce que veut dire la CSC en citant un « objectif lié à la santé ». Les recours futurs devant les tribunaux posant la même question que celle au cœur de l'affaire Cuthbertson contre Rasouli seront et devront toujours être tranchés de la même façon que dans l'affaire Cuthbertson contre Rasouli. Hawryluck et coll. font également une erreur d'interprétation quant au rôle de la Commission du consentement et de la capacité (CCB) de l'Ontario. L'examen et la compréhension des principes éthiques médicaux en jeu fourniront un cadre dans lequel évoluer.

La norme de soins décrit les pratiques médicales usuelles de médecins prudents à un moment donné et dans des circonstances particulières. C'est une opinion de 
la majorité qui s'affirme devant les tribunaux, mais qui se relâche au chevet du patient. La norme de soins et l'obligation du médecin ont toujours penché fermement en faveur du maintien de la vie. La norme de soins peut-elle, à elle seule, constituer une raison suffisante pour justifier l'interruption d'un traitement? Il est faux de suggérer que si la CSC avait considéré la norme de soins, elle aurait été à la hauteur et aurait soutenu les médecins. La Cour n'a jamais concédé que seuls les médecins peuvent décider de l'étendue de l'expertise médicale. Le tribunal a le pouvoir de statuer sur des questions sur lesquelles il estime avoir le pouvoir de le faire et l'a déjà fait savoir. Dans l'affaire Walker Estate contre York Finch General Hospital et autres, ${ }^{2}$ la CSC a déterminé qu'un dépliant de la Croix-Rouge qui était remis aux donneurs de sang potentiels pour décourager les dons par des groupes à haut risque d'infection par le VIH était inadéquat en dépit des témoignages contraires apportés par deux experts médicaux. Cette affaire a montré que la Cour ne permettrait pas à des médecins de prendre des décisions sur des questions qui ne nécessitaient pas de compétences spéciales ou une expertise particulière. Si un problème dépasse les connaissances des tribunaux, ceux-ci se fieront à l'avis des experts, mais il est présomptueux de suggérer que la question de savoir si une vie vaut la peine d'être vécue repose sur l'avis d'un expert médical. Seul l'individu lui-même connait la valeur de sa propre vie.

En utilisant le terme « Objectif lié à la santé », la CSC souligne que la législature de l'Ontario a cherché à clarifier le fait qu'un consentement était nécessaire pour n'importe quel traitement. Un objectif lié à la santé affirme sans aucune ambiguité que l'arrêt d'un traitement constitue un traitement et que tous les traitements nécessitent un consentement. Si c'est la capacité de donner un consentement qui est problématique, le CCB est l'endroit adapté pour résoudre les conflits sur le consentement. À l'origine, le CCB a été créé pour passer en revue les internements involontaires au titre de la Loi sur la santé mentale. $^{\mathrm{A}}$ Le conseil du CCB est traditionnellement constitué de juristes, de profanes et d'un psychiatre. Dans le cas d'un internement involontaire, la présence d'un psychiatre au sein du conseil est nécessaire pour agir à la place du psychiatre traitant qui a déclenché la procédure d'internement involontaire. Dans le cas d'un conflit sur la fin de vie déclenché par un médecin, et lorsqu'aucun substitut du preneur de décision n'existe, le conseil joue alors le rôle du patient jusqu'à donner le consentement à l'arrêt du système de maintien des fonctions vitales. Ajouter au conseil des médecins formés aux soins intensifs, comme le suggèrent Hawryluck et coll. revient à ne pas comprendre le rôle du CCB. Le CCB ne se préoccupe pas des normes d'exercice médical et il n'a pas à en savoir plus que n'en saurait un profane en la matière. Un médecin ne peut pas décider de façon unilatérale d'ignorer une décision prise par un patient compétent ou son substitut pour la prise de décision sur des questions en rapport avec l'autonomie, y compris de l'arrêt de traitement. Si un groupe de médecins s'entend sur un traitement et le propose comme norme de soins, cela ne s'impose pas plus au patient que si un seul médecin l'avait recommandé. Les normes de soins n'ont jamais été destinées à devenir une tactique d'appel au peuple (un argumentum ad populum) pour forcer un patient, réticent mais compétent au sens juridique, à s'y conformer.

Un argument couramment avancé pour justifier l'arrêt des systèmes de maintien des fonctions vitales concerne les souffrances impossibles à traiter. La souffrance est liée au concept de "bénéfice ", un mot provenant du latin benefactum qui signifie littéralement un «bien fait», une bonne action. Une bonne action n'est pas une action unilatérale. Un « bénéfice » est décidé par contrat; il doit y avoir une offre, une acceptation et une réflexion. Le patient doit accepter que l'accord est en fait «bon » et autonomie signifie que le patient décide de ce à quoi il peut se conformer ou non. Dans le cas de la souffrance, la norme de soins devient une question de valeurs. Bien que nous puissions être d'accord sur le fait que nous sommes contre la souffrance, les présomptions de la présence de souffrance avancées par les observations externes de tierces parties ne peuvent pas être objectives car la souffrance est toujours subjective, et donc relative. La souffrance est souvent liée au concept de futilité; toutefois, les arguments de futilité sont une tautologie et il n'y a pas de consensus sur les détails. N'essayons pas de prétendre que les médecins sont objectifs face à l'arrêt des systèmes de maintien des fonctions vitales. En réalité, la norme de soins est loin d'être monolithique. La prise de décision d'un médecin n'est pas exempte de biais et des études ont montré que les décisions de fin de vie des médecins dépendent souvent des convictions religieuses de ces derniers et de la zone géographique dans laquelle ils exercent. $^{3}$

Les principes seuls ne font que guider les théories éthiques défendables; ils ne les gouvernent pas. La vertu de prendre soin des autres peut libérer le médecin des concepts étroits contenus dans les codes de déontologie. La pratique de la médecine au chevet du patient est un équilibre entre notre raison et notre désir. Qu'est-ce que les patients attendent le plus de nous? Le concept aristotélicien de phroneis, ou de la sagesse pratique, s'applique ici. Les médecins ayant du discernement savent non seulement qu'un principe s'applique, mais aussi comment l'appliquer dans les circonstances qui se présentent à eux. Le médecin expérimenté crée un lien de confiance avec le patient et la confiance guide le traitement. Les patients et les familles adoptent rarement des positions qui défient la raison. Mais 
quand la confiance est brisée, chaque désaccord devient insurmontable. Il faut éviter d'en arriver là. On doit savoir faire marche arrière, c'est-à-dire prendre un «temps mort », laisser les émotions se calmer et revenir étudier les recommandations. Les décisions de fin de vie sont les décisions les plus importantes que prennent les individus. Nous devons laisser aux patients le temps dont ils ont besoin. Les spécialistes de soins intensifs savent que chacun est confronté à la mort en dépit de nos efforts. Quand une famille consent à un arrêt de traitement, ce n'est pas une victoire de la norme de soins, comme Hawryluck et coll. voudraient qu' on le comprenne, et si cela prend plus de temps aux patients ou à ceux qui décident à leur place pour accepter le caractère inévitable de la mort, alors qu'il en soit ainsi.

Conflicts of interest None declared.
Conflit d'intérêt Aucun déclaré.

\section{References}

1. Hawryluck L, Baker AJ, Faith A, Singh JM. The future of decisionmaking in critical care after Cuthbertson v. Rasouli. Can J Anesth 2014; 61: this issue. DOI:10.1007/s12630-014-0215-9.

2. Walker Estate v. York Finch General Hospital. Supreme Court Judgment. April 19, 2001; SCR 647. Available from URL: http:// scc-csc.lexum.com/scc-csc/scc-csc/en/item/1859/index.do (accessed July 2014).

3. Sprung CL, Cohen SL, Sjokvist P, et al. End-of-life practices in European intensive care units: the Ethicus Study. JAMA 2003; 290: 790-7. 